
\title{
25 Research Soure \\ Ultralow-dose Pediatric Total-body PET/CT Imaging Using an Artificial Intelligence Technique
}

\section{Qiyang Zhang}

Shenzhen Institutes of Advanced Technology Chinese Academy of Sciences https://orcid.org/0000-00016185-3080

\section{Zizheng Xiao}

Sun Yat-sen University Cancer Center

\section{Xu Zhang}

Sun Yat-sen University Cancer Center

\section{Yingying Hu}

Sun Yat-sen University Cancer Center

\section{Yumo Zhao}

Sun Yat-sen University Cancer Center

\section{Jingyi Wang}

United Imaging Healthcare Group

\section{Yun Zhou}

United Imageing Healthcare Group

\section{Hairong Zheng}

Shenzhen Institutes of Advanced Technology Chinese Academy of Sciences

\section{Wei Fan}

Sun Yat-sen University Cancer Center

\section{Dong Liang}

Shenzhen Institutes of Advanced Technology Chinese Academy of Sciences

\section{Zhanli Hu ( $\square$ zl.hu@siat.ac.cn )}

Shenzhen Institutes of Advanced Technology Chinese Academy of Sciences

\section{Research Article}

Keywords: positron emission tomography, machine learning, pediatric, low-dose, total-body

Posted Date: February 1st, 2022

DOI: https://doi.org/10.21203/rs.3.rs-1304664/v1

License: (c) (1) This work is licensed under a Creative Commons Attribution 4.0 International License. Read Full License 


\section{Abstract}

\section{Purpose}

Young children are more sensitive to radiation than adults, and their absorption of effective dose can be four times higher than that of adults, inducing a higher risk of secondary injury. Here, we propose for the first time the use of artificial intelligence techniques combined with low dose CT prior information to improve image quality in ultralow-dose total-body PET/CT scans.

\section{Methods}

A total of 44 pediatric patients (weight range: $8 \cdot 5-50 \cdot 1 \mathrm{~kg}$; ages $1-12$ years) who underwent total-body $\mathrm{PET} / \mathrm{CT}$ at the Sun Yat-sen University Cancer Center were retrospectively enrolled. ${ }^{18} \mathrm{~F}-\mathrm{FDG}$ was administered at a dose of $3.7 \mathrm{MBq} / \mathrm{kg}$ and an acquisition of $600 \mathrm{~s}$. The low-dose PET images were simulated by truncating the list-mode data to reduce the count density. The neural network uses the residual network as the basic structure and fuses low-dose CT images as the priori information into the network at different scales. The image quality was assessed by subjective and objective analyses. Bland-Altman plots were used to assess the agreement of regional SUV ratios between the image types. Statistical analysis was carried out to assess the differences in the image quality metrics and reader agreement.

\section{Results}

The use of artificial intelligence techniques can significantly improve PET image quality. When combined with a prior CT information, the anatomical information of the images was better recovered, and the 15 seconds acquisition yields a quality equivalent to the 10 minutes acquisition, it can equivalently guide the concentration of the injected tracer to decrease, which is very important for dose-sensitive pediatric patients.

\section{Conclusions}

The proposed artificial intelligence technology is safe and can effectively enhance the quality of pediatric total-body PET/CT ultralow-dose images and has the potential to further reduce the concentration of injected tracers for clinical applications.

\section{Introduction}

Positron emission tomography (PET) combined with computed tomography (CT), referred to as PET/CT, is an indispensable malignancy diagnostic device in hospital radiology departments $(1-4)$. The occurrence of malignant tumor is not localized, but often a systemic disease(5-7). Therefore, PET-CT generally takes whole-body scan, which can discover not only the primary site lesion, but also the presence of metastatic lesions in soft tissue organs and bones in various parts of the body, which is very helpful for the staging of tumor and determining the scope of metabolically active lesions, and provides accurate information on the site of puncture or tissue biopsy. It provides more reasonable and accurate positioning for radiation therapy (especially precision radiotherapy) and reduces the side effects of treatment(4). PET uses radioactive tracers, special cameras and computers to image tracer distribution and evaluate organ and tissue functions. 
Typically, the tracer administration activity and event signal acquisition time are positively correlated with the imaging quality $(8,9)$. However, highly active tracers will increase the risk of secondary cancer in patients, and longer acquisition times may introduce autonomic or involuntary patient motion artifacts into the images. Therefore, the activity of radiotracer administration and the time duration of data collection are often restricted by radiation safety and tolerability $(3,10-12)$. Compared with adults, children are more sensitive to ionizing radiation, and the effective dose absorbed can reach 4-5 times that of adults(10). Pediatric patients who are exposed to radiation at an early stage have a higher risk of developing malignant tumors because their bodies are developing and their life expectancy is longer $(11,13,14)$. In addition, the lack of self-control ability in children is also a problem. The PET data acquisition process is relatively time-consuming compared to $\mathrm{CT}$. The movement of the child during the event signal acquisition will cause image artifacts, can blur the image, and cause difficulty in making the diagnosis(15). Therefore, in pediatric nuclear medicine, it is important to minimize the dose of the radiotracer that is administered as well as the acquisition time of the event signal.

The typical PET axial field-of-view (FOV) is $20 \mathrm{~cm}$, and when performing a whole-body PET scan, data needs to be collected from multiple locations, over $85 \%$ of the body at each location lies outside the scanner's FOV, and signals from these areas of the body cannot be collected, yielding less than $1 \%$ sensitivity to the signal, making it very difficult to achieve an overall dose reduction $(16,17)$. Ultra-long axial FOV is regarded as a new generation of PET technology that can fully improve signal sensitivity(17). Recently, a long-axis FOV PET scanner was introduced $(3,18,19)$. This PET scanner, called uEXPLORER (uEXPLORER, United Imaging Healthcare, Shanghai, China), has an axial FOV of $194 \mathrm{~cm}$ and can record coupled photons from the entire body simultaneously, hence the name total-body PET imaging. It increases the effective sensitivity by a factor of approximately 40 with respect to the $20 \mathrm{~cm}$ axial FOV, thus allowing conventional image quality to be achieved using lower injection concentrations and shorter acquisition times $(11,16,20)$. Based on this platform, artificial intelligence techniques are used to explore the low-dose limit of rapid scanning achievable by current PET devices, which is of clinical importance, especially for pediatric PET diagnosis.

In this retrospective study of pediatric data, we aim to use artificial intelligence (Al) techniques (CNNs, convolutional neural networks) to further perform ultralow-dose image recovery on a total-body PET/CT system that already has the advantage of using a low dose. CNNs based on multimodal data fusion have been shown to combine the advantages of the data of each modality, which can effectively and significantly reduce the dose(21-24). We will use the accompanying CT images as a prior knowledge to enhance the anatomical information of the images. The enhanced synthetic network we adopted takes the residual module as the main framework, introduces the high-dimensional information of the prior CT at different scales, and uses the perceptual loss to ensure the effective restoration of the structure, and uses the simulated annealing training strategy to speed up the training process(25-27). The experimental results show that the CNN can effectively synthesize images from the ultralow-dose images and can reach the clinical diagnosis level, and the network model has an improved performance in the anatomical structure recovery with the introduction of CT prior image information.

\section{Materials And Methods}


The data of this retrospective study came from the Sun Yat-sen University Cancer Center. The study was approved by the institutional review board of this center, and informed consent was obtained from all of the patients' legal guardians.

\section{Data Acquisition}

A total of 44 pediatric patients who underwent total-body PET/CT using the uEXPLORER scanner at the Sun Yat-sen University Cancer Center from July 2020 to August 2020 were retrospectively enrolled in this study. The inclusion criteria were as follows: (1) age $<13$ and (2) body weight $<60 \mathrm{~kg}$, and the exclusion criteria were (1) waiting time after 18F-FDG injection $\geq 75 \mathrm{~min}$, (2) diffuse liver involvement, and (3) no suspected FDGavid lesion.

A K-fold cross-validation strategy (here $\mathrm{K}=5$ ) was used to compensate for the lack of training samples, and after training, we selected the last set of 5 patients ( 4 males, 1 female) as the evaluation set (Table 1). The dose of $18 \mathrm{~F}-\mathrm{FDG}$ was approximately $3.7 \mathrm{MBq} / \mathrm{kg}$, and the acquisition time was $600 \mathrm{~s}$. Low-dose total-body CT scans were acquired at a tube current of $10 \mathrm{~mA}$ and voltage of $100 \mathrm{kV}$ (rotation time $0.5 \mathrm{~s}$, pitch 1.0125, collimation $80 \times 0.5 \mathrm{~mm}$ ) and were reconstructed in a $512 \times 512$ matrix for attenuation correction. Low-dose PET images $(0.037-0.925 \mathrm{MBq} / \mathrm{kg})$ were simulated by truncating the data in the list mode (equivalent to compressing the event signal sampling time) to reduce the count density.

The acquisition time of PET images was $600 \mathrm{~s}$, and PET images were reconstructed for $600,150,60,30,15$, and $6 \mathrm{~s}$ to simulate five dose levels (Figure 2): $0.93 \mathrm{MBq} / \mathrm{kg}$ (1/4 dose, G150s), $0.37 \mathrm{MBq} / \mathrm{kg}(1 / 10$ dose, G60s), $0.19 \mathrm{MBq} / \mathrm{kg}$ (1/20 dose, G30s), $0.09 \mathrm{MBq} / \mathrm{kg}$ (1/40 dose, G15s), and 0.04 MBq/kg (1/100 dose, G6s). All PET images were reconstructed using the time-of-flight ordered subset expectation maximization method (TOF-OSEM) with a set of parameters: PSF modeling, 3 iterations, 20 subsets, matrix $256 \times 256$, slice thickness $2.89 \mathrm{~mm}$, pixel size $2.34 \times 2.34 \times 2.89 \mathrm{~mm} 3$, Gaussian postfiltering $(3 \mathrm{~mm})$, and all necessary correction methods, including scattering and attenuation corrections.

TABLE1

Patient characteristics

\begin{tabular}{|lllll|}
\hline Patient characteristics & \multicolumn{3}{l}{ Total Data Set } & \multicolumn{2}{l|}{ Test Data Set } \\
\cline { 2 - 5 } & Mean \pm SD & Range & Mean \pm SD & Range \\
\hline Ages (years) & $5 \cdot 9 \pm 3 \cdot 4$ & $1 \cdot 0-12 \cdot 0$ & $4 \cdot 4 \pm 2 \cdot 7$ & $1 \cdot 0-8 \cdot 0$ \\
\hline Weight $(\mathrm{kg})$ & $20 \cdot 7 \pm 10 \cdot 2$ & $8 \cdot 5-50 \cdot 1$ & $15 \cdot 7 \pm 5 \cdot 6$ & $10-24 \cdot 3$ \\
\hline Height (cm) & $113 \cdot 7 \pm 23 \cdot 6$ & $70 \cdot 0-162 \cdot 0$ & $101 \cdot 0 \pm 17 \cdot 4$ & $75 \cdot 0-124 \cdot 0$ \\
\hline Injected dose (MBq) & $78 \cdot 5 \pm 40$ & $32 \cdot 9-184 \cdot 6$ & $61 \cdot 1 \pm 21$ & $39 \cdot 6-93 \cdot 6$ \\
\hline Injected dose per weight (MBq/kg) & $3 \cdot 8 \pm 0 \cdot 27$ & $2 \cdot 4-4 \cdot 2$ & $3 \cdot 9 \pm 0 \cdot 06$ & $3 \cdot 83-3 \cdot 96$ \\
\hline Waiting time $(\mathrm{min})$ & $63 \cdot 9 \pm 7$ & $48-78$ & $62 \cdot 8 \pm 9 \cdot 6$ & $52-73$ \\
\hline Lesion size $(\mathrm{mm})$ & $8 \cdot 1 \pm 2$ & $5-19$ & $8 \pm 1 \cdot 3$ & $5-16$ \\
\hline
\end{tabular}




\section{Image Preprocessing}

The CT images were registered to the PET images using MATLAB (MathWorks, Natick, MA) software after considering the position offset of the patient in different acquisition modes. All images were resampled to the voxel dimensions of the acquired PET volumes. The PET image pixel values were converted to SUV, and the $\mathrm{CT}$ image pixel values were approximated as the values of the absorption coefficients of water to $\mathrm{X}$-ray at $60 \mathrm{keV}$. A circular mask was created and applied to the PET and registered CT images based on the maximum torso diameter of 44 patients and the World Health Organization (WHO) Child Growth Standards(28). The processed PET images and CT images were used as input into the CNN.

\section{CNN Implementation}

The proposed neural network for low-dose PET image synthesis is shown in Figure 1. Based on an investigative assessment of different state-of-the-art deep learning structures, including ResNet(25) and UNet(27), we used the U-Net encoder-decoder architecture strategy with the residual module as the main framework to introduce the prior CT information at different scales into the network. The fusion from the high-dimensional features of the individual modal images can lead to better integration of complementary information in each modality $(26,29)$. Therefore, we used the high-dimensional features extracted from the $\mathrm{CT}$ images after $\mathrm{N}$ convolutional layers (here, $\mathrm{N}=3,5$ ) as the prior information introduced into the encoder of the network.

The inputs to the network were the low-dose PET images and low-dose CT images. The full-dose PET image was treated as the ground truth. To enhance the network's ability to recover anatomical structures and texture details, the loss function used a combination of L2 normal and perceptual loss(30). The network was constructed using the PyTorch deep learning framework and was optimized using the Adam optimizer with a cosine annealing strategy to speed up convergence $(31,32)$. We open sourced the network code so that readers can obtain detailed information on the parameters such as the learning rate and batch size from the code(33).

\section{Quantitative Imaging Analysis}

The objective image quality evaluation was performed by an experienced technician under the supervision of a radiologist. The images generated by the neural network were first visually inspected for artifacts. For each axial section, the image quality of the synthetic PET images and the original low-dose PET images were compared to the full-dose images using the peak signal-to-noise ratio (PSNR) and the structural similarity index (SSIM). Two-dimensional circular regions of interest (ROIs) with a diameter of $2 \mathrm{~cm}$ were drawn over a homogeneous area of the liver parenchyma, being careful to avoid blood vessels and tumors to record semiquantitative uptake measurements of the liver, such as the SUVmax and SUVmean. The shortest lengthdiameter of the 18F-FDG-avid suspected lesion (not necessarily malignant) was identified and the ROls were drawn for this lesion on the section with the largest lesion diameter to record semiquantitative uptake measurements of the lesion location, including the SUVmax and SUVmean.

\section{Qualitative Imaging Assessment}


A subjective assessment of the PET image quality was rated independently by two nuclear radiologists (a senior radiologist with $>10$ years of experience and a radiologist with $>5$ years of experience) based on a 5point Likert scale. The synthesized PET images, the low-dose PET images, and the full-dose PET images of each data set were anonymized. The 5-point Likert scale was used for (1) the overall impression of the image quality, (2) the conspicuity of the major suspected malignant lesions, (3) the conspicuity of the organ anatomical structures and (4) the image noise. The status read from the full-dose images was treated as the ground truth. For each PET image, the physician assigned an image quality score on a five-point scale: 1 , uninterpretable; 2, poor; 3, adequate; 4, good; and 5, excellent. Examples of images of grades 1-5 are illustrated in Figure 2 (a-e).

\section{Statistical Analysis}

The statistical analysis was performed with the R Statistical package and Microsoft Excel. Cohen's kappa test was used to measure the interrater agreement of the subjective scores between the raters. Kappa values of $0 \cdot 41-0.60,0.61-0.80$, and $0 \cdot 81-1 \cdot 00$ indicated moderate, substantial, and excellent agreement, respectively. Tukey's honestly significant difference test was used for multiple comparisons when comparing the subjective image quality scores between the groups. A paired t-test was conducted to compare the objectivity of the image quality metrics between the different dose groups for different image processing methods. The SSIM, PSNR, SUVmean and SUVmax were calculated. $\mathrm{p}<0.05$ was considered statistically significant (Bonferroni correction was used to account for multiple comparisons when necessary).

\section{Results}

\section{Image Quality}

The objective measurements of the image quality are shown in Figures 3-5. The average SSIM and PSNR values that were calculated from the synthesized images and low-dose images relative to the full-dose images for all the patients in the evaluation set are shown in Figure 3. The synthesized images show marked improvement in the image quality compared with the low-dose images. The image synthesized from the lowdose PET combined with the CT prior model had the highest structural similarity index and the highest peak signal-to-noise ratio in the data set of all dose groups. The image quality metrics and the $P$ value of the paired t-test showed that deep learning could significantly suppress the noise in the low-dose images (G6s$G 60 s, P<0.05$ ), but it was not significant for the relatively high-dose PET images (G150s, $P>0 \cdot 05$ ). The structural similarity was also significantly improved (G6s-G150s, $P<0.05$ ). The addition of the prior CT information significantly improved the effect of the deep learning methods in processing the low-dose images (G6s-G30s, P<0.05), while the improvement was not significant for the relatively high-dose PET images (G60s-G150s, $P>0 \cdot 05$ ). All the methods showed that there were significant differences between the different dose groups $(P<0 \cdot 05)$ (Supplementary Table $S 1)$.

The semiquantitative uptake measurements obtained from the uniform areas of the liver parenchyma, SUVmax and SUVmean, are presented in Table 2. The standard deviation of the SUVmean and SUVmax values of low-dose PET decreased with an increasing dose, while that of the images synthesized by artificial intelligence did not change much, were more stable and could reach the standard of reference images in the 
lowest dose case. To further compare the differences between the two artificial intelligence methods, a Bland-Altman plot was drawn (Figure 5), which showed that the Al-based method combined with the prior synthesized CT images had the lowest bias and almost the lowest variance among all of the dose groups compared to the reference standard full-dose images.

TABLE 2 The average SUVmean and SUVmax with standard deviations in the healthy liver

\begin{tabular}{|llllll|}
\hline $\begin{array}{l}\text { Liver } \\
\text { SUVmean }\end{array}$ & G6s & G15s & G30s & G60s & G150s \\
\hline $\begin{array}{l}\text { Low Dose } \\
\text { PET }\end{array}$ & $0.9197 \pm 0.2750$ & $0.9125 \pm 0.1585$ & $0.9096 \pm 0.1264$ & $0.9288 \pm 0.1058$ & $0.9235 \pm 0.0742$ \\
$\begin{array}{l}\text { Synthesized } \\
\text { PET }\end{array}$ & $0.9445 \pm 0.0699$ & $0.9120 \pm 0.0471$ & $0.9011 \pm 0.0494$ & $0.9100 \pm 0.0562$ & $0.9088 \pm 0.0520$ \\
\hline $\begin{array}{l}\text { Synthesized } \\
\text { PET } \sim \text { Prior }\end{array}$ & $0.9101 \pm 0.0555$ & $0.8985 \pm 0.0471$ & $0.9070 \pm 0.0469$ & $0.9181 \pm 0.0526$ & $0.9113 \pm 0.0517$ \\
\hline $\begin{array}{l}\text { All data were presented as the } \\
\text { mean } \pm \text { SD }\end{array}$ & & Reference Full dose PET (G600s): $0.9045 \pm 0.0555$ \\
\hline
\end{tabular}

\begin{tabular}{|lcllll|}
\hline $\begin{array}{l}\text { Liver } \\
\text { SUVmax }\end{array}$ & G6s & G15s & G30s & G60s & G150s \\
\hline $\begin{array}{l}\text { Low Dose } \\
\text { PET }\end{array}$ & $1 \cdot 8377 \pm 0 \cdot 3917$ & $1 \cdot 3553 \pm 0 \cdot 1803$ & $1 \cdot 3177 \pm 0 \cdot 1085$ & $1 \cdot 2411 \pm 0 \cdot 1446$ & $1 \cdot 1113 \pm 0 \cdot 1151$ \\
\hline $\begin{array}{l}\text { Synthesized } \\
\text { PET }\end{array}$ & $1 \cdot 1150 \pm 0 \cdot 1771$ & $1 \cdot 0010 \pm 0 \cdot 1182$ & $0 \cdot 9943 \pm 0 \cdot 1202$ & $1 \cdot 0428 \pm 0 \cdot 1187$ & $1 \cdot 0273 \pm 0 \cdot 1207$ \\
\hline $\begin{array}{l}\text { Synthesized } \\
\text { PET } \sim \text { Prior }\end{array}$ & $1 \cdot 0485 \pm 0 \cdot 1426$ & $0 \cdot 9820 \pm 0 \cdot 1110$ & $1 \cdot 0231 \pm 0 \cdot 1133$ & $1 \cdot 0430 \pm 0 \cdot 1169$ & $1 \cdot 0340 \pm 0 \cdot 1181$ \\
$\begin{array}{l}\text { All data were presented as the } \\
\text { mean } \pm \text { SD }\end{array}$ & & Reference Full dose PET (G600s): $1 \cdot 0138 \pm 0 \cdot 1300$ \\
\hline
\end{tabular}

Figure 4 shows the SUV change measurements at the lesion position in a patient with bilateral cervical mediastinal lymphoma. The changes from G6s, 15 s, G30s, G60s, and G150s to G600s were calculated by subtracting the respective measurements from G600s. The change in the metrics gradually decreased with an increasing dose. The SUV change of the image synthesized by artificial intelligence integrated with CT prior information was the smallest in all dose cases.

Table 3 shows the paired t-tests for the changes in SUVmean and SUVmax relative to the full-dose images between the images of the different methods for each dose group. The changes in metrics (SUVmean and SUVmax) synthesized by the artificial intelligence methods were significantly different from the low-dose images in all of the dose groups $(P<0.05)$. The difference between the two artificial intelligence methods was significant under the SUVmean metric $(P<0.05)$, while the difference was not significant with an increasing 
dose under the SUVmax metric (G60s - G150s, p>0.05). The artificial intelligence method combined with the prior CT information had an advantage at lower doses.

TABLE 3 Paired t-tests for the changes in SUVmean and SUVmax relative to the full-dose images between the images of the different methods for each dose group.

\begin{tabular}{|llllll|}
\hline SUVmean(P Value) & G6s & G15s & G30s & G60s & G150s \\
\hline (LD-REF) vs. (SP-REF) & 0.000 & 0.000 & 0.000 & 0.000 & 0.000 \\
\hline (LD-REF) vs. (SP P-REF) & 0.000 & 0.000 & 0.000 & 0.000 & 0.000 \\
\hline (SP-REF) vs. SP P & 0.000 & 0.000 & 0.000 & 0.000 & 0.002 \\
\hline
\end{tabular}

\begin{tabular}{|lccccc|}
\hline SUVmax(P Value) & G6s & G15s & G30s & G60s & G150s \\
\hline (LD-REF) vs. (SP-REF) & 0.000 & 0.000 & 0.000 & 0.000 & 0.000 \\
\hline (LD-REF) vs. (SP P-REF) & 0.000 & 0.000 & 0.000 & 0.000 & 0.001 \\
\hline (SP-REF) vs. (SP P-REF) & 0.005 & 0.013 & 0.033 & 0.146 & 0.242 \\
\hline $\begin{array}{l}\text { LD: Low Dose PET SP: Synthesized PET } \\
\text { SP P: Synthesized PET Prior REF: Reference Full dose PET }\end{array}$ \\
\hline
\end{tabular}

\section{Clinical Readings}

The subjective image quality scores of the different methods were compared in the different dose groups, where the mean \pm SD of the Likert scores for the overall image metrics are presented in Table 4. The radar plot of all perspectives (significance of major suspicious malignant lesions, significance of organ anatomy and image noise) is shown in Figure 6. Synthetic PET with the prior CT information method had an excellent performance for all perspectives in the different dose groups.

The interrater agreement of the Likert scores is important when performing subjective studies of the images. For each of the three methods (low-dose PET, synthesized PET and synthesized PET combined with prior) the Cohen's kappa values for (1) the overall impression of image quality, (2) the significance of major suspicious malignant lesions, (3) the significance of the organ anatomy and (4) the image noise are listed in Table 5. For the G6s dose group, the kappa coefficient for the low-dose PET was 0.5045 , indicating a moderate degree of agreement between the two radiologists. Additionally, the kappa coefficient for synthesized PET was $0 \cdot 7713$, indicating a high degree of agreement between the two radiologists. For the other cases, the agreement between the two radiologists was almost perfect (0·8383-1·0).

The results of Tukey's honestly significant difference test comparing the subjective image quality scores between the groups are shown in Supplementary Table S2-S3. Significant differences were found in the image scores that were synthesized by the artificial intelligence methods for the different dose groups 
compared to the original low-dose image scores in (1) the overall impression of image quality, (2) the significance of major suspicious malignant lesions, (3) the significance of organ anatomy and (4) the image noise. There were almost no significant differences between the two Al methods (Supplementary Table S2). There was no significant difference in (4) the image noise between the different dose groups of the same method. For (2) the significance of major suspicious malignant lesions, there were significant differences between the low-dose groups (G6s-G30s, G6s-G60s, G6s-G150s) and no significant differences between the high-dose groups. Significant differences existed for (3) the significance of organ anatomy except for G60sG50S (Supplementary Table S3).

TABLE 4 Subjective image quality as assessed by the 5-point Likert scale

\begin{tabular}{|c|c|c|c|c|c|}
\hline Likert scoring & G6s & G15s & G30s & G60s & G150s \\
\hline Low Dose PET & $1 \cdot 00 \pm 0 \cdot 000$ & $1 \cdot 167 \pm 0 \cdot 389$ & $2 \cdot 000 \pm 0 \cdot 426$ & $2 \cdot 917 \pm 0 \cdot 515$ & $4 \cdot 000 \pm 0 \cdot 603$ \\
\hline Synthesized PET & $2 \cdot 167 \pm 0 \cdot 577$ & $2 \cdot 833 \pm 0.937$ & $4 \cdot 000 \pm 0 \cdot 603$ & $4 \cdot 917 \pm 0 \cdot 289$ & $5 \cdot 000 \pm 0 \cdot 000$ \\
\hline Synthesized PET Prior & $2 \cdot 250 \pm 0 \cdot 452$ & $3 \cdot 500 \pm 0 \cdot 522$ & $4 \cdot 500 \pm 0 \cdot 522$ & $5 \cdot 0 \pm 0 \cdot 000$ & $5 \cdot 0 \pm 0 \cdot 000$ \\
\hline
\end{tabular}

TABLE 5 Cohen's kappa values for low-dose PET, synthetic PET and synthetic PET combined with CT prior methods under four metrics: (1) the overall impression of image quality, (2) the significance of major suspicious malignant lesions, (3) the significance of organ anatomy and (4) the image noise-

\begin{tabular}{|lllll|}
\hline Cohen's kappa & $\begin{array}{l}\text { Overall image } \\
\text { quality }\end{array}$ & $\begin{array}{l}\text { Lesion } \\
\text { Conspicuity }\end{array}$ & $\begin{array}{l}\text { Anatomy } \\
\text { Conspicuity }\end{array}$ & $\begin{array}{l}\text { Image } \\
\text { Noise }\end{array}$ \\
\hline Low Dose PET & 0.5045 & 0.9104 & 1.0000 & 0.9134 \\
\hline Synthesized PET & 0.7713 & 0.8383 & 0.8613 & 1.0000 \\
\hline $\begin{array}{l}\text { Synthesized PET } ~ \\
\text { Prior }\end{array}$ & 0.9523 & 1.0000 & 0.9511 & 1.0000 \\
\hline
\end{tabular}

\section{Discussion}

The ultralong axial FOV $(2 \mathrm{~m})$ total-body PET/CT platform allows for high-quality imaging at lower tracer doses and shorter acquisition times and is well suited for pediatric patients who are dose sensitive and have difficulty cooperating with prolonged examinations. Our aim was to further investigate ultralow-dose imaging on the total-body PET/CT platform using artificial intelligence techniques to evaluate the shortest data acquisition time that can be allowed on this platform when standard doses are used (the equivalent lowest injected tracer dose when the standard acquisition lengths are used, or both the shortest data acquisition time and the lowest injected tracer dose). 
This proof-of-concept study shows that the use of artificial intelligence techniques can be effective in improving the quality of low-dose images. Compared to the low-dose PET images and the images synthesized by a model that does not consider the use of CT images as a prior information, the images synthesized by a network model that considers the prior have higher average image quality and lower regional SUV relative value bias and variance. This indicates the important value of introducing CT images with rich anatomical structure information into the imaging model.

The quantitative results, such as the data shown in Figures 2-5 and Tables 2-3, show that the combined performance of image quality becomes progressively higher as the acquisition time increases and that the images synthesized by the model using CT images as a priori information have quantitative values that are closest to those of the full-dose reference images (SSIM, PSNR) for the same duration. Table 2 and Figure 45 also show that the stability of the SUV values can be more effective, which can ensure the accuracy when performing a PET quantitative analysis.

The qualitative results, such as the results shown in Figure 6 and Tables 4-5, show that the images synthesized based on Al techniques performed best in terms of the image noise. In the 15-second data case, the images synthesized by the network model using CT prior information excelled in terms of the anatomical structure, which indicates that $\mathrm{CT}$ anatomical structure information plays a key role. In the 30 -second data case, the Al-based images achieved the standard of the full-dose images in almost all aspects.

There are several limitations to our study. A total of 44 pediatric data of different ages and genders were used in this retrospective study, and $\mathrm{K}$-fold cross validation was used to compensate for the lack of training samples to improve the generalizability of the network model. Although there was no overfitting of the network on the test set, the lack of real clinical samples may have overfitting for the application of the model in new cases, so a more accurate network model needs to be obtained after collecting more samples for training. Our study is based on a conventional ${ }^{18} \mathrm{~F}-\mathrm{FDG}$ injection protocol that may not extrapolate to tracers other than ${ }^{18} \mathrm{~F}-\mathrm{FDG}$, such as ${ }^{18} \mathrm{~F}-\mathrm{NaF},{ }^{18} \mathrm{~F}-\mathrm{FET},{ }^{68} \mathrm{Ga}$-PSMA, etc. Due to limited data on small lesions, a lesion detection rate subgroup analysis was not performed for different lesion sizes. More studies are needed to investigate the effect of the lesion shape, volume, etc. on the detection rates. The method fully applies the data obtained from PET/CT scans with different modalities and explores the acquisition time limit. The current work in this paper only does low-dose studies for PET imaging, and the CT prior images are used for images acquired by the scanning device under low-dose loading conditions. Since CT brings much more radiation than PET in the whole PET/CT scan, targeted reduction of CT modal dose can bring greater benefits to patients, especially pediatric patients. Later, we will use artificial intelligence technology to conduct a study on dual-modality dose reduction of PET/CT.

\section{Conclusion}

Based on all quantitative and qualitative results, we can see that the enhancement of total-body PET/CT ultra-low dose images using artificial intelligence techniques can significantly improve image quality and thus can guide the reduction of injected tracer concentration, which has a very promising potential for clinical diagnostic applications in dose-sensitive pediatric patients. 


\section{Declarations}

\section{Funding}

This work was supported by the National Natural Science Foundation of China (32022042, 81871441, 62001465), the Shenzhen Excellent Technological Innovation Talent Training Project of China (RCJC20200714114436080), the Natural Science Foundation of Guangdong Province in China (2020A1515010733), and the Chinese Academy of Sciences Key Laboratory of Health Informatics in China (2011DP173015). Jingyi Wang, Jiatai Feng and Yun Zhou are employees of the United Imaging Healthcare group. No other potential conflict of interest relevant to this article was reported.

\section{Author Contributions}

Qiyang Zhang ${ }^{1,2,3^{*}}$, Zizheng Xiao ${ }^{4,}{ }^{*}$, Xu Zhang ${ }^{4}$, Yingying $\mathrm{Hu}^{4}$, Yumo Zhao ${ }^{4}$, Jingyi Wang ${ }^{5}$, Yun Zhou ${ }^{5}$, Hairong Zheng ${ }^{1,2}$, Wei Fan ${ }^{4}$, Dong Liang ${ }^{1,2}$, Zhanli Hu ${ }^{1,2 \#}$

Zhanli Hu conceptualised and designed the study, coordinated the study, analysed the data, and reviewed and revised the manuscript. Xu Zhang, Yingying Hu and Yumo Zhao collected the data, analysed the data and revised the manuscript. Qiyang Zhang and Zizheng Xiao drafted the initial manuscript, analysed the data, carried out statistical analysis, and reviewed and revised the manuscript. Hairong Zheng, Wei Fan and Dong Liang helped to conceptualise and design the study, coordinated and supervised data collection, collected data, and reviewed and revised the manuscript. Jingyi Wang and Yun Zhou interpreted the data, analysed the data, and reviewed and revised the manuscript. All authors approved the final manuscript as submitted and agree to be accountable for all aspects of the work.

\section{Data Availability}

The datasets generated during and/or analyzed during the current study are available from the corresponding author on reasonable request.

\section{Ethics approval}

All procedures performed in studies involving human participants were in accordance with the ethical standards of the institutional and/or national research committee and with the 1964 Helsinki declaration and its later amendments or comparable ethical standards.

\section{Consent to participate}

Informed consent was obtained from legal guardians.

\section{Consent for publication}

Additional informed consent was obtained from all legal guardians for whom identifying information is included in this article. 
Jingyi Wang, Jiatai Feng and Yun Zhou are employees of the United Imaging Healthcare group. The other authors have no conflicts of interest.

\section{References}

1. Townsend D. Dual-Modality Imaging: Combining Anatomy and Function*. Journal of Nuclear Medicine. 2008;49:938 - 955.

2. Boellaard R, Delgado-Bolton R, Oyen W, et al. FDG PET/CT: EANM procedure guidelines for tumour imaging: version 2.0. European Journal of Nuclear Medicine and Molecular Imaging. 2014;42:328 - 354.

3. Zhang X, Cherry S, Xie Z, Shi H-c, Badawi R, Qi J. Subsecond total-body imaging using ultrasensitive positron emission tomography. Proceedings of the National Academy of Sciences of the United States of America. 2020;117:2265 - 2267.

4. von Schulthess GK, Steinert HC, Hany TF. Integrated PET/CT: current applications and future directions. Radiology. 2006;238 2:405-422.

5. Salazar H, Godwin AK, Daly MB, et al. Microscopic benign and invasive malignant neoplasms and a cancer-prone phenotype in prophylactic oophorectomies. Journal of the National Cancer Institute. 1996;88 24:1810-1820.

6. Qian B, Pollard JW. Macrophage Diversity Enhances Tumor Progression and Metastasis. Cell. 2010;141:39-51.

7. Liotta LA, Steeg PS, Stetler-Stevenson WG. Cancer metastasis and angiogenesis: An imbalance of positive and negative regulation. Cell. 1991;64:327-336.

8. Wahl RL. Principles and Practice of PET/CT 2/e. Lippincott Williams \& Wilkins. 2008.

9. Shreve P, Shreve P, Fachgebiete H. Clinical PET-CT in Radiology. Springer New York. 2011.

10. Stauss J, Franzius C, Pfluger T, et al. Guidelines for 18F-FDG PET and PET-CT imaging in paediatric oncology. European Journal of Nuclear Medicine and Molecular Imaging. 2008;35:1581-1588.

11. Zhao Y-M, Li YH, Chen T, et al. Image quality and lesion detectability in low-dose pediatric 18F-FDG scans using total-body PET/CT. European Journal of Nuclear Medicine and Molecular Imaging. 2021:1 8.

12. Huang B, Law M, Khong P. Whole-body PET/CT scanning: estimation of radiation dose and cancer risk. Radiology. 2009;251 1:166-174.

13. Fahey FH, Goodkind A, MacDougall RD, et al. Operational and dosimetric aspects of pediatric PET/CT. Journal of Nuclear Medicine. 2017;58:1360-1366.

14. Treves S, Gelfand M, Parisi M, et al. Update: Image Gently and Nuclear Medicine at 10 Years. Journal of nuclear medicine : official publication, Society of Nuclear Medicine. 2019;60 4:7N-9N.

15. Nadel H, Shulkin B. Pediatric positron emission tomography-computed tomography protocol considerations. Seminars in ultrasound, CT, and MR. 2008;29 4:271-276.

16. Tan $\mathrm{H}$, Sui $\mathrm{X}$, Yin $\mathrm{H}$, et al. Total-body PET/CT using half-dose FDG and compared with conventional PET/CT using full-dose FDG in lung cancer. European Journal of Nuclear Medicine and Molecular Imaging. 2020;48:1966 - 1975. 
17. Cherry SR, Jones TL, Karp JS, Qi J, Moses W, Badawi RD. Total-Body PET: Maximizing Sensitivity to Create New Opportunities for Clinical Research and Patient Care. The Journal of Nuclear Medicine. 2018;59:12 - 13.

18. Badawi R, Shi H-c, Hu P, et al. First Human Imaging Studies with the EXPLORER Total-Body PET Scanner*. The Journal of Nuclear Medicine. 2019;60:299 - 303.

19. Zhang X, Xie Z, Berg E, et al. Total-Body Dynamic Reconstruction and Parametric Imaging on the uEXPLORER. The Journal of Nuclear Medicine. 2020;61:285 - 291.

20. Zhang Y-q, Hu P, Wu R, et al. The image quality, lesion detectability, and acquisition time of 18F-FDG total-body PET/CT in oncological patients. European Journal of Nuclear Medicine and Molecular Imaging. 2020;47:2507 - 2515.

21. Xiang L, Qiao Y, Nie D, et al. Deep auto-context convolutional neural networks for standard-dose PET image estimation from low-dose PET/MRI. Neurocomputing. 2017;267:406-416.

22. Chen K, Gong E, Macruz FBdC, et al. Ultra-Low-Dose 18F-Florbetaben Amyloid PET Imaging Using Deep Learning with Multi-Contrast MRI Inputs. Radiology. 2019;290 3:649-656.

23. Gao J, Li P, Chen Z, Zhang J. A Survey on Deep Learning for Multimodal Data Fusion. Neural Computation. 2020;32:829-864.

24. Hosseini M-P, Tran TX, Pompili D, Elisevich KV, Soltanian-Zadeh H. Multimodal data analysis of epileptic EEG and rs-fMRI via deep learning and edge computing. Artificial intelligence in medicine. 2020;104:101813.

25. He K, Zhang X, Ren S, Sun J. Deep Residual Learning for Image Recognition. 2016 IEEE Conference on Computer Vision and Pattern Recognition (CVPR). 2016:770-778.

26. Y. L, Y. B, G. H. Deep Learning. Nature. 2015;521:436-444.

27. Ronneberger O, Fischer P, Brox T. U-Net: Convolutional Networks for Biomedical Image Segmentation. Paper presented at: MICCAI, 2015.

28. WHO. https://www.who.int/.

29. Kumar A, Fulham M, Feng D, Kim J. Co-Learning Feature Fusion Maps From PET-CT Images of Lung Cancer. IEEE Transactions on Medical Imaging. 2020;39:204-217.

30. Johnson J, Alahi A, Fei-Fei L. Perceptual Losses for Real-Time Style Transfer and Super-Resolution. ArXiv. 2016;abs/1603.08155.

31. Kingma DP, Ba J. Adam: A Method for Stochastic Optimization. CoRR. 2015;abs/1412.6980.

32. Loshchilov I, Hutter F. SGDR: Stochastic Gradient Descent with Warm Restarts. arXiv: Learning. 2017.

33. https://gitee.com/codedomain/low-dose_pet_synn.git [computer program]. Version; 2021.

\section{Figures}




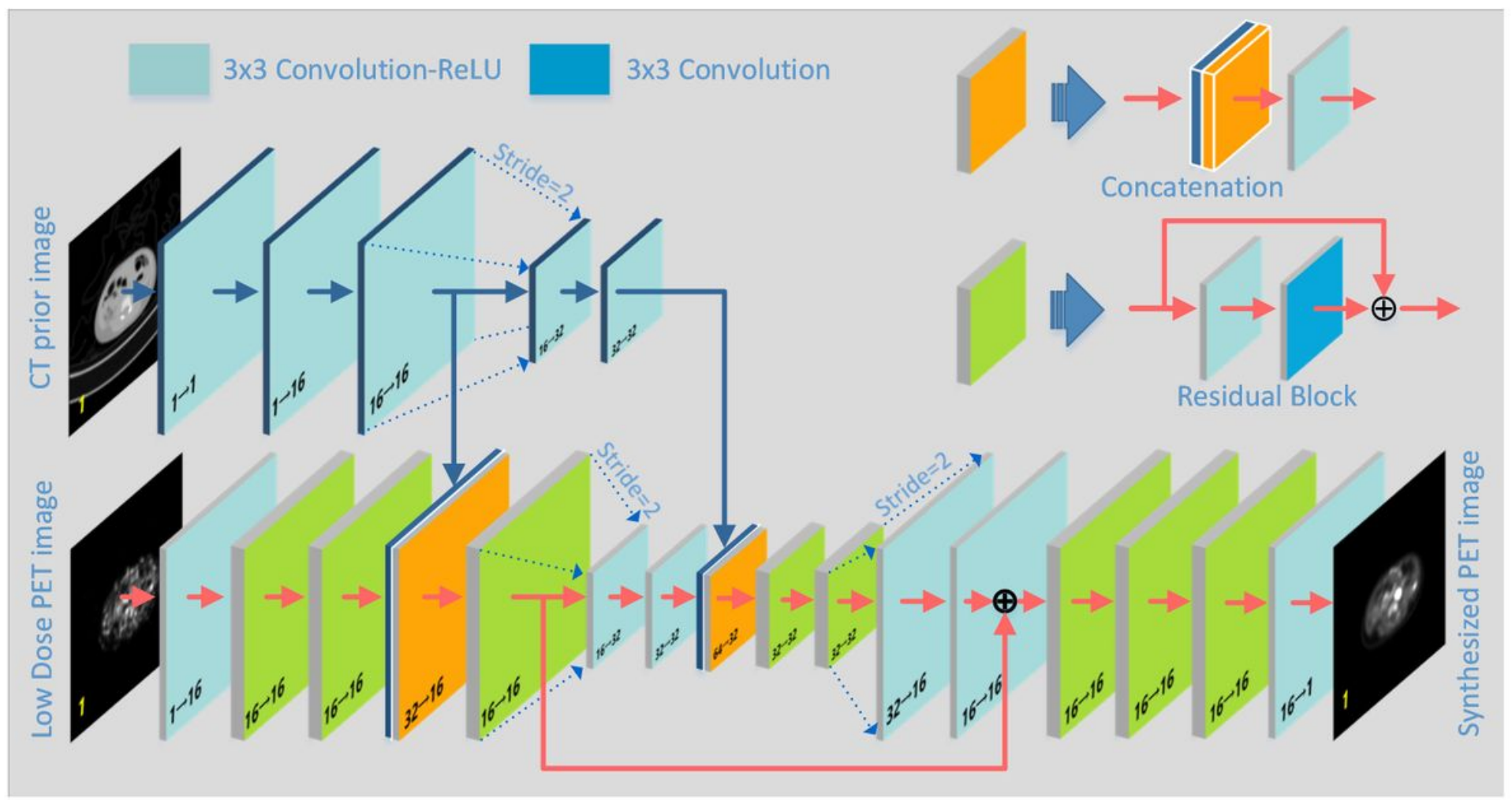

\section{Figure 1}

Schematic diagram of the convolutional neural network used in this work.

It includes two modality-specific encoders and one decoder that synthesizes the full-dose PET images. The arrows indicate the flow of computational operations, and the lower left corner of the box is labeled with the number of input and output feature images for this module. 


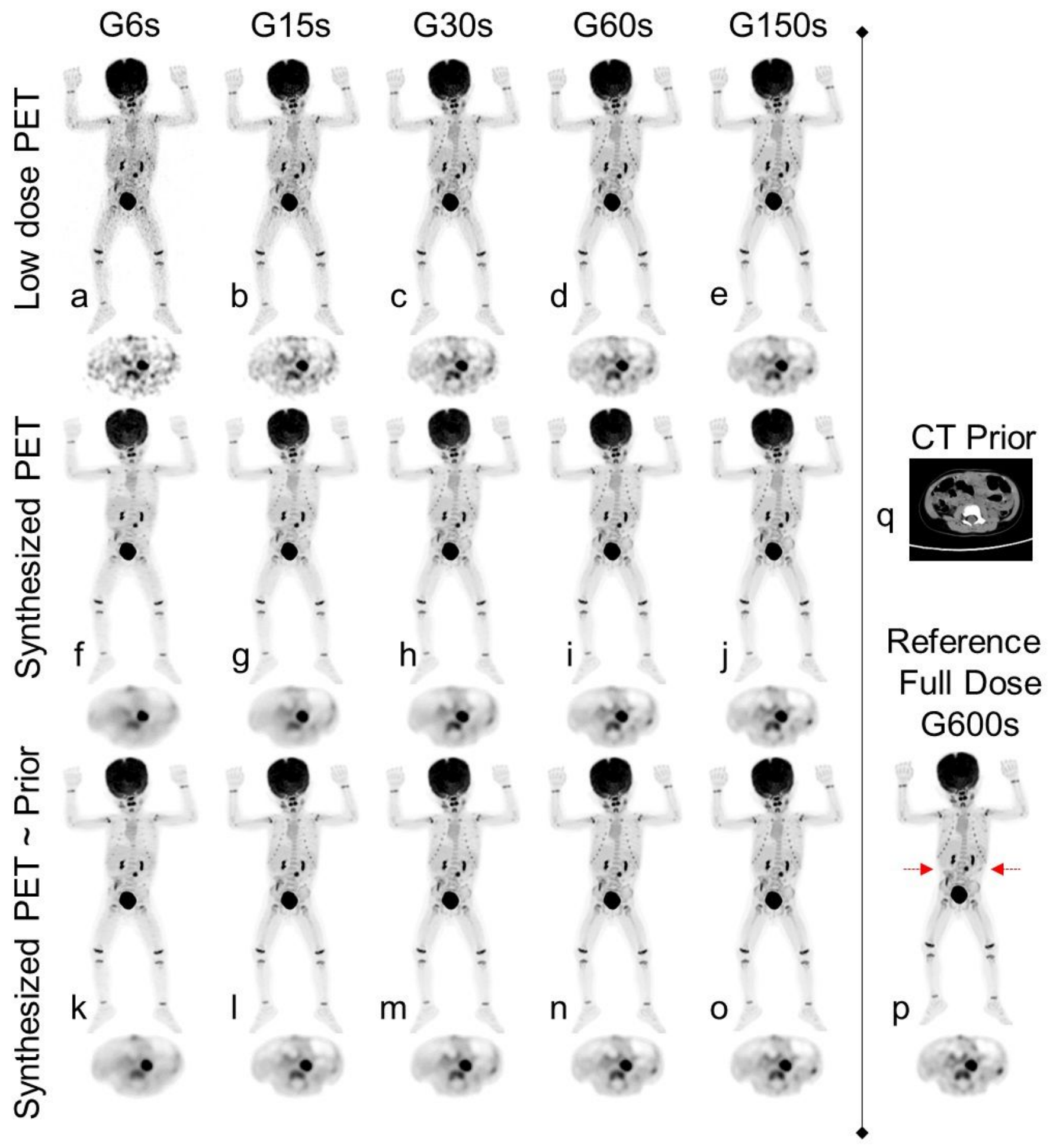

Figure 2

${ }^{18} \mathrm{~F}$-FDG PET images of a 2-year-old male patient weighing $10 \mathrm{~kg}$ with metabolic activity in the left abdominal wall after surgery for a left testicular yolk sac tumor.

(a-e) The low-dose PET images were reconstructed into 6 (G6s), 15 (G15s), 30 (G30s), 60 (G60s), and 150 (G150s) seconds and are shown in MIP and axial views. (f-j) The MIP and axial view PET images synthesized from a-e using CNN. (k-0) The MIP and axial view PET images synthesized from a-e using CNN 
combined with the prior CT information. (p) Reference, full dose image with the axis view locations marked. (q) The prior CT image. The synthesized PET images show significantly reduced noise compared to the lowdose PET images, and the images generated from the PET combined with the prior CT information model were superior in reflecting the underlying anatomic patterns compared with the images generated from the PET-only model.

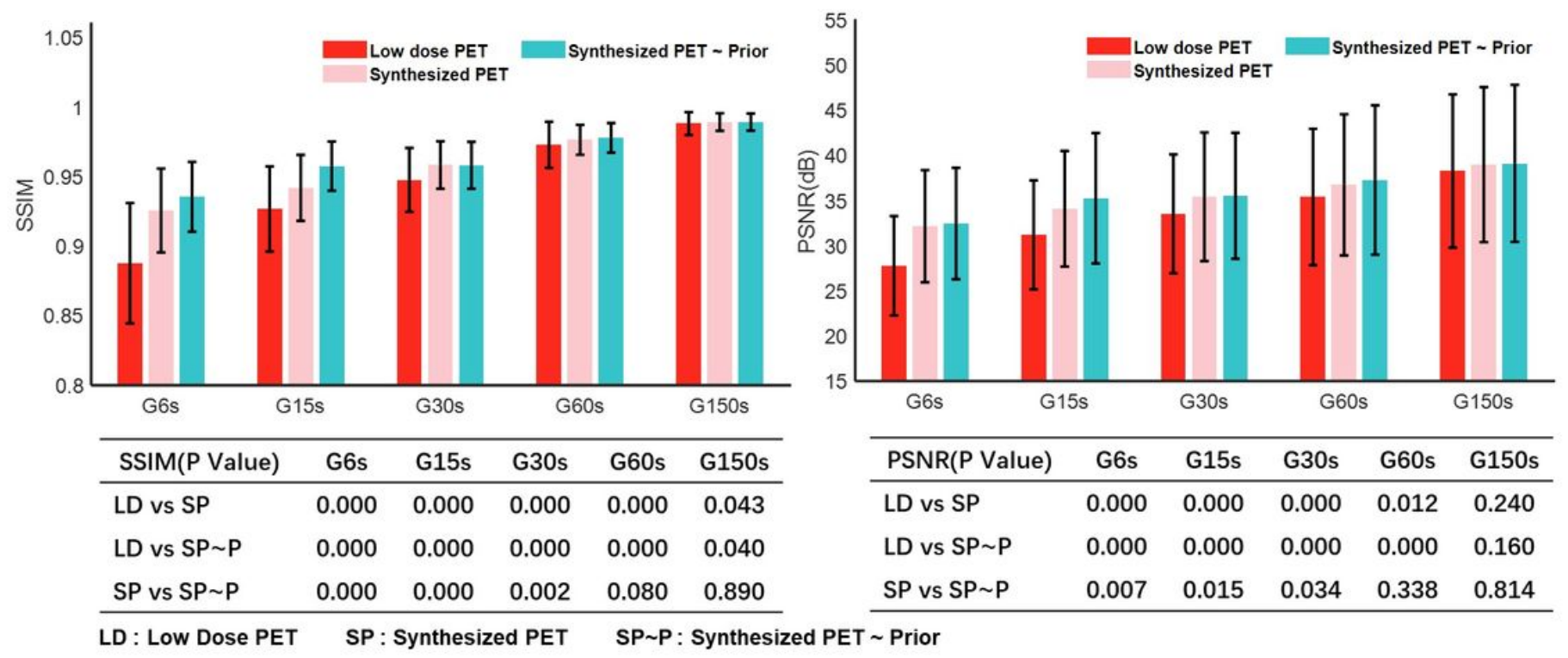

Figure 3

Image quality metrics comparing the images from low-dose PET, the synthesized PET-only model, and the synthesized PET combined with CT prior model.

For all of the metrics, the comparison were to the full-dose PET images. The images from the synthesized PET with the CT prior model were superior for all of the metrics, including a higher structural similarity index (SSIM) and a higher peak signal-to-noise ratio (PSNR). 


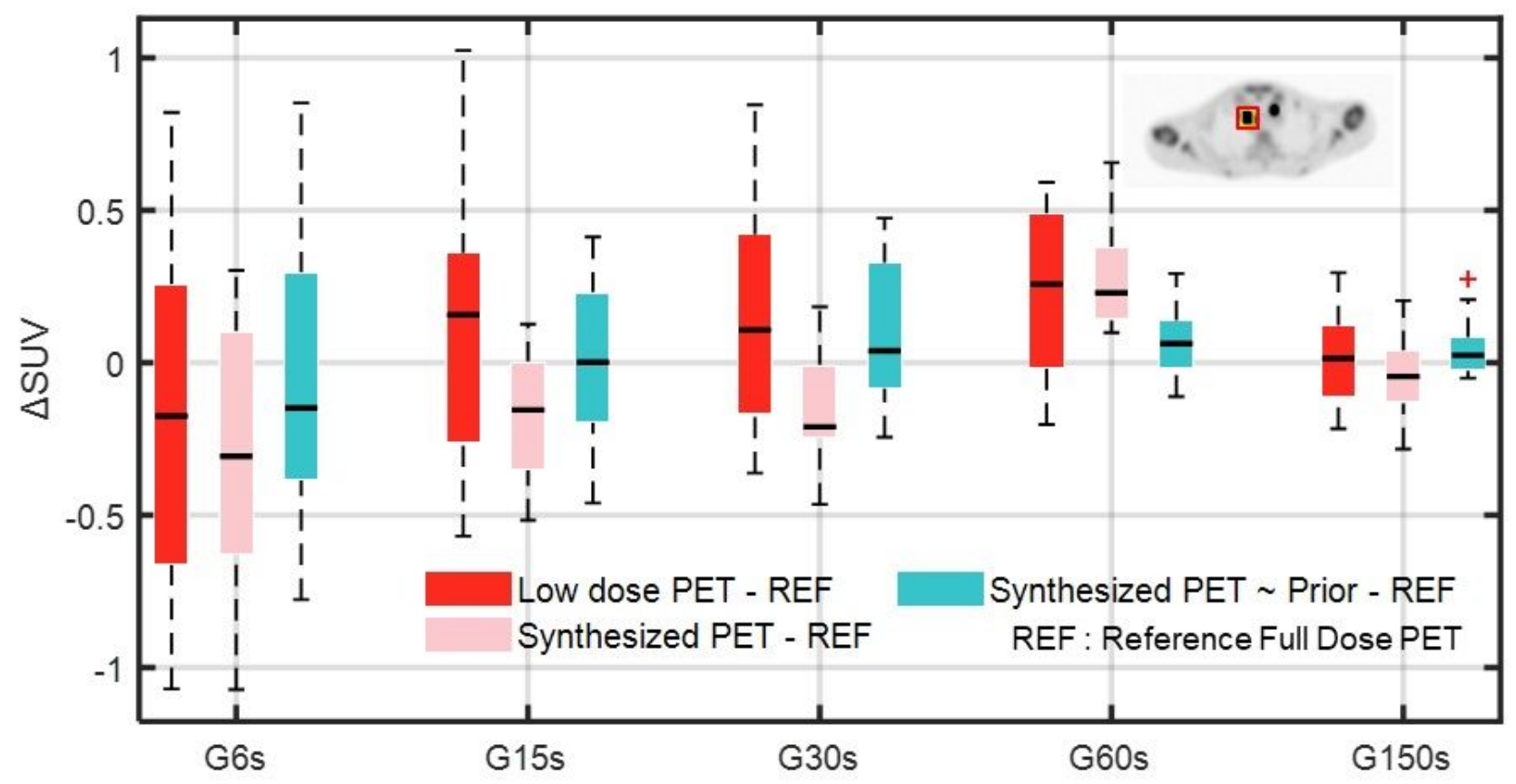

Figure 4

Boxplot of the SUV changes of lesion locations in a patient with bilateral cervical mediastinal lymphoma.

The change in each metric was calculated by subtracting the respective measurements from G600s. The changes in the metrics gradually decreased as the dose was increased. The SUV change of the image synthesized by artificial intelligence that integrates the prior CT information is the smallest in all the dose cases. 


\section{Low Dose PET}

- REF
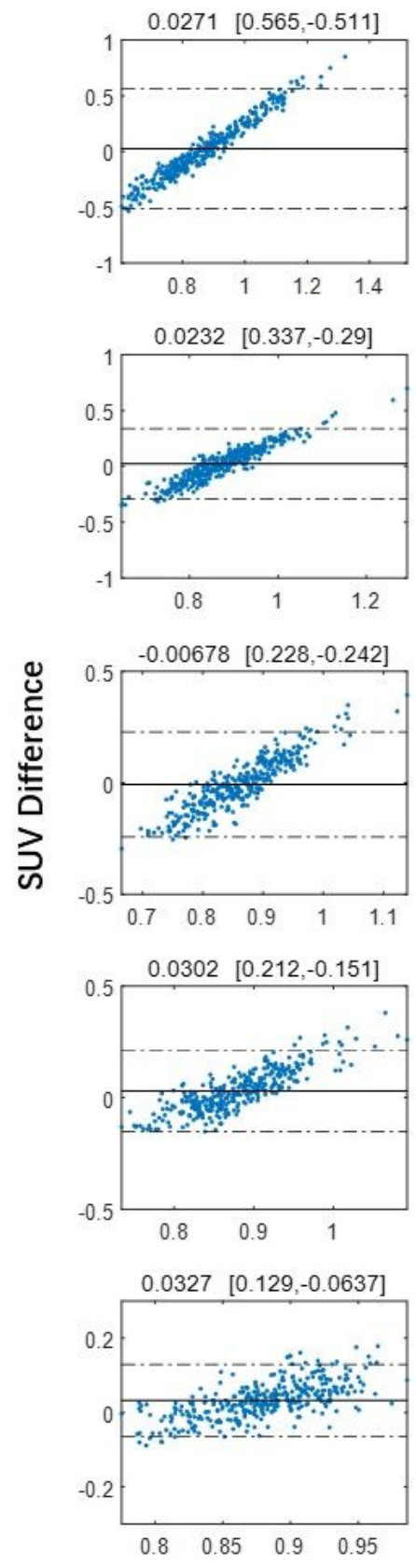

Synthesized PET -

REF
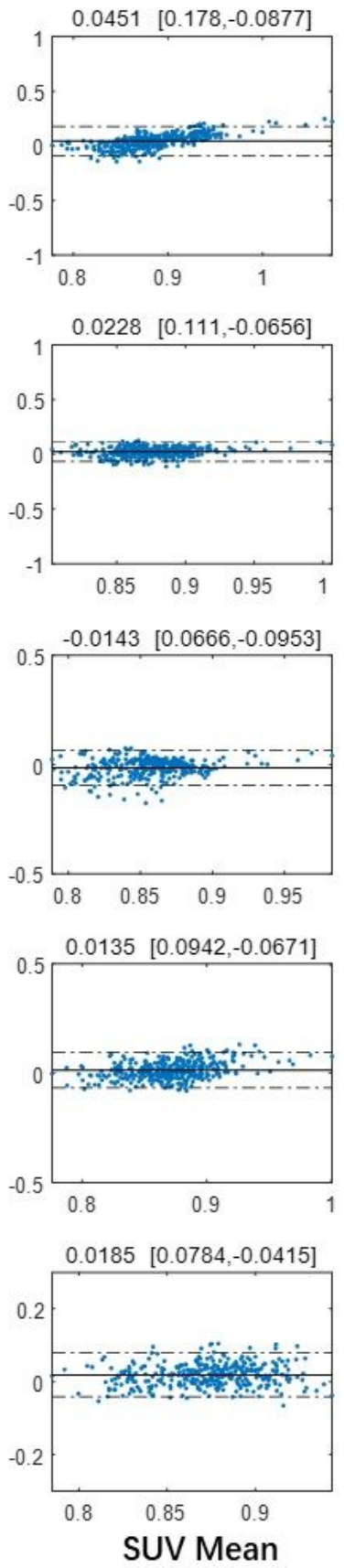

Synthesized PET Prior -

REF
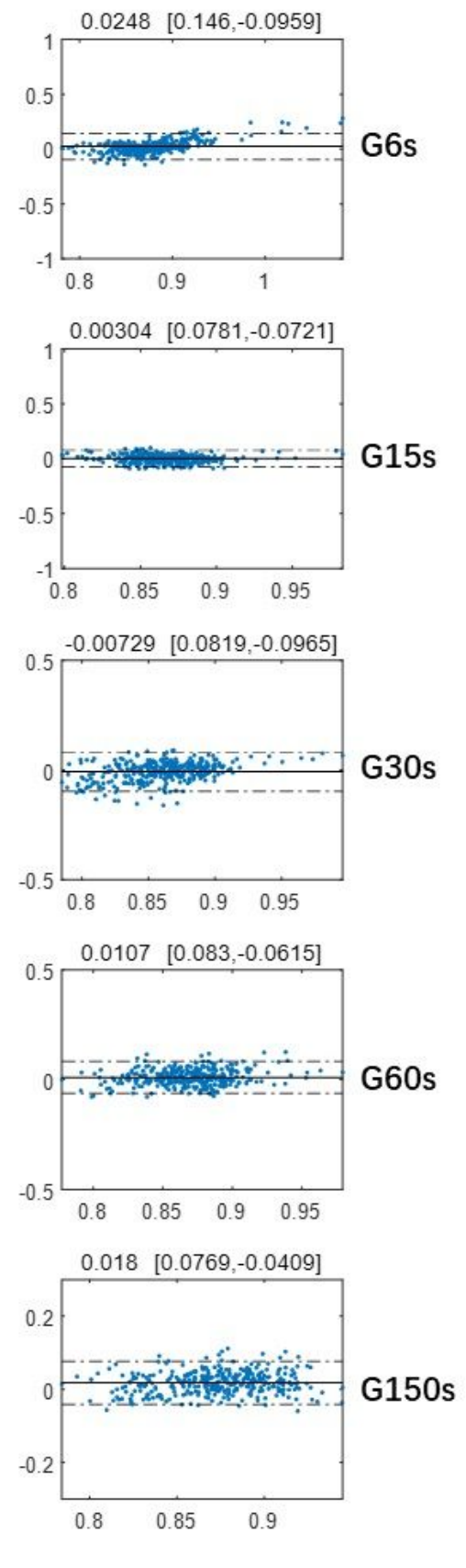

\section{Figure 5}

\section{Bland-Altman analysis of SUV differences compared between methods (low-dose PET, synthetic PET,}

synthetic PET combined with the CT prior) and references across all of the data sets.

The solid and dashed lines denote the mean and 95\% confidence interval (CI) of the SUV differences, respectively. The images synthesized from the synthesized PET combined with the prior model had the lowest bias and had almost the smallest $95 \% \mathrm{Cl}$ relative to the reference full-dose images. 

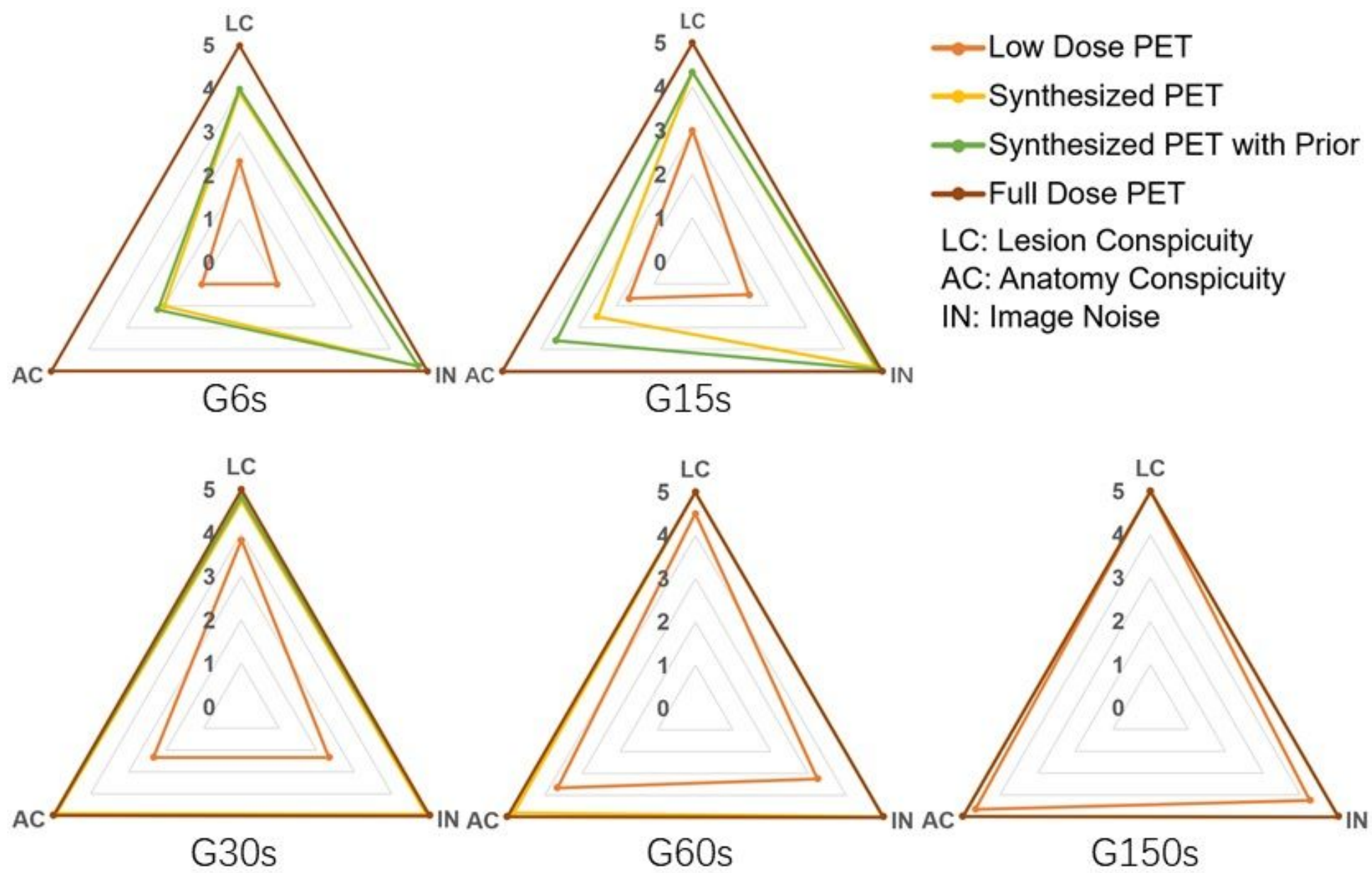

Figure 6

Radar plots of the subjective image quality scores for the different methods in the different dose groups, including the lesion conspicuity scores, anatomy conspicuity scores, and image noise scores.

\section{Supplementary Files}

This is a list of supplementary files associated with this preprint. Click to download.

- GraphicalAbstract.pdf

- Supplementary.pdf 